\title{
Los caminos hacia una enseñanza no tradicional de la química
}

Paths toward a non-traditional teaching of chemistry

\section{Volumen 18, Número 2}

Mayo-Agosto

pp. 1-20

Este número se publica el 1 de mayo de 2018

DOI: https://doi.org/10.15517/aie.v18i2.33164

Gabriel José Ordaz González

Maj Britt Mostue

Revista indizada en REDALYC, SCIELO

Revista distribuida en las bases de datos:

LATINDEX, DOAJ, REDIB, IRESIE, CLASE, DIALNET, SHERPA/ROMEO, QUALIS-CAPES, MIAR

Revista registrada en los directorios:

ULRICH'S, REDIE, RINACE, OEI, MAESTROTECA, PREAL, CLACSO 


\title{
Los caminos hacia una enseñanza no tradicional de la química
}

Paths toward a non-traditional teaching of chemistry

\author{
Gabriel José Ordaz González 1 \\ Maj Britt Mostue ${ }^{2}$
}

\begin{abstract}
Resumen: Es preocupante cómo todavía hay docentes (de química) que piensan que la enseñanza es llenar con conocimientos a quienes serían "contenedores vacíos", es decir, los estudiantes. Posiblemente, esta concepción tradicional-expositiva asumida en la praxis pedagógica contribuye a que la enseñanza de la química esté (o se mantenga) en crisis. El propósito de este ensayo es presentar algunas reflexiones sobre el rol del docente de química a partir de una mejor conceptualización de la enseñanza y del aprendizaje que la ofrecida por la educación tradicional. Esto, como un recordatorio y un llamado de atención para que las personas docentes asuman los retos en la enseñanza de esta ciencia. Una educación, concebida para promover la autonomía en el aprendizaje y la metacognición, obliga al profesorado de química a "enseñar" una ciencia más contextualizada, desde un pensamiento multinivel y con criticismo. Lo anterior, podría contribuir a un mejor entendimiento del conocimiento químico y coadyuvar en los procesos formativos.
\end{abstract}

Palabras clave: modelo educacional, enseñanza de la química, competencias del docente, formación.

Abstract: It is worrying how still there are teachers (of chemistry) that think teaching means to fill up with knowledge the "empty brains" of students. Possibly, this traditional-expositive approach assumed by those teachers in their pedagogical praxis contributes chemistry teaching is (or keep) in crisis. The aim of this essay is to present some reflections about the role of chemistry teachers according to better conceptualizations of teaching and learning than the offered by traditional education, as a reminder and a call of attention to them to assume the challenges in chemistry teaching. A teaching conceived to promote the autonomy in the learning and the metacognition obligates chemistry teachers to "teach" a science more contextualized, since the multilevel thought and with criticism. This could contribute to a better chemical knowledge understanding, and help the formative processes.

Key words: educational model, chemistry education, competencies of teacher, formation.

\footnotetext{
${ }_{1}$ Profesor Asistente del Departamento de Química, Escuela de Ciencias, Universidad de Oriente, Núcleo de Sucre, Venezuela. Magister Scientiarum en Educación mención Enseñanza de la Química.Dirección electrónica: gordaz@udo.edu.ve

2 Profesora Asociada del Departamento de Química, Escuela de Ciencias, Universidad de Oriente, Núcleo de Sucre, Venezuela.
}

Ensayo recibido: 10 de noviembre, 2017

Enviado a corrección: 02 de abril, 2018

Aprobado: 23 de abril, 2018 


\section{Introducción}

En la enseñanza formal de aula, en especial de la química, los estudiantes se enfrentan constantemente a nuevos lenguajes, concepciones abstractas y procedimientos matemáticos que podrían resultarles confusos, requiriendo un gran esfuerzo cognitivo para lograr una comprensión de esos contenidos. Aunado a ello, traerlos al escenario académico desde la instrucción (o exposición) parece ya no ser suficiente.

El esquema tradicional de enseñanza, vertical y unidireccional, parece aislar tanto al docente como al estudiante en dinámicas educativas totalmente diferentes. Por un lado, el docente apuesta por contenidos predispuestos en un currículo, muchas veces presentados de forma descontextualizada, que deben ser igualmente asimilados por todos los estudiantes tal cual son dados, desconociendo las individualidades de los procesos formativos. El estudiante, por su parte, se esfuerza en la apropiación ingenua de esos contenidos, no para la constitución de saberes útiles en su devenir y realidad, sino como elementos temporales que le permitirán cumplir con las tareas académicas asignadas, especialmente en pro de una calificación, la promoción a un siguiente nivel, o un título escolar, que lo envuelve en una constante presión psicosocial de "fracaso" o "éxito".

Es decir, el docente siente cumplida su tarea al "creer que trasmite" sus conocimientos de los temas del currículo a sus estudiantes, juzgando luego las capacidades de éstos para asimilar o no dichos contenidos en momentos específicos con una actividad en particular (generalmente, un examen escrito). Mientras, el estudiante se limita a escuchar/ver pasivamente su clase, sintiendo cumplida su tarea con su asistencia o memorizando transitoriamente algo, sin comprenderlo, que le permitirá realizar la actividad que lo califica (quizá, juzgando luego y en silencio las capacidades del docente para "transmitir" esos conocimientos que cree debió "recibir").

Estos dos escenarios no parecen ser consistentes con un real proceso educativoformativo. Por ello, esta postura tradicional ha sido ampliamente criticada (Arias y Peralta, 2011; Eschenhagen, 2007; Freire, 2005; Gavidia, 2005; López, 2008; Molina, 2006; Pérez, 2002). Aun así, parece mantenerse y extenderse en muchos centros de enseñanza en todos los niveles, especialmente al enseñar ciencias, como es el caso de química.

La idea del presente ensayo es plantear algunos elementos de reflexión sobre cuál debe ser la labor del docente, especialmente en la enseñanza de la química, más allá de una exposición ciega de contenidos. 


\section{2. ¿A qué llamamos enseñanza y aprendizaje? ¿Procesos aislados o sinonímicos?}

Una discusión necesaria, de la que depende cómo abordamos el proceso pedagógico, es nuestra conceptualización de los términos enseñanza y aprendizaje. Éstos han sido vislumbrados, generalmente, bajo una figura dicotómica, entendiéndose como algo consecuencial, es decir, bajo la línea direccional de esa falsa transmisión de contenidos. Esto también se ve reflejado en la relación de los roles del docente, quien "enseña", y del estudiante, a quien se "enseña" (aun cuando lo correcto sería, quien "aprende").

De acuerdo con Álvarez Gallego (2003) el término enseñanza vino a sustituir otros más restringidos de la visión tradicional como el de instrucción, tratando de humanizar un poco el proceso educativo. Sin embargo, si en la praxis se sigue asumiendo la enseñanza como instrucción, más que constituirla como un proceso, seguirá asociándose con la actividad particular de mostrar, dictar o exponer, en la que el docente es el "poseedor" de aquel conocimiento que debe ser "transmitido" a los estudiantes. El aprendizaje, por su parte, también seguirá concibiéndose como la "recepción", "adquisición" y/o "acumulación" de esos conocimientos proporcionados por el docente. Aunque, hoy es sabido que el conjunto de conocimientos expuestos al impartir una clase "no son apropiados por [el estudiante] en tanto tales sino que son enfrentados como meros contenidos posibles de ser re-construidos y así ser trans-formados en un nuevo conocimiento" (Molina, 2006, p. 56).

En otras palabras, una clase expositiva solo permite traer al escenario pedagógico una serie de informaciones, pero, para que un estudiante pueda o no procesarlas y darles significado debe valerse de una gama de aspectos que lo constituyen como ser biológico, histórico, cultural y social (intereses, imaginación, experiencias, conocimientos previos, entre muchos otros). Se puede pensar que ha habido una evolución de las ideas sobre lo que es el aprendizaje, pasando de ser un simple proceso de adquisición/acumulación de conocimientos, con manifiestos cambios de conducta, a uno más complejo, de construcción de conocimientos, mediado en un ambiente social y cultural, que implica cambios en las estructuras cognitivas del estudiante.

Ahora bien, si reconocemos que el aprendizaje es un proceso autónomo, lo cual, como indica Escribano, "implica por parte del que aprende asumir la responsabilidad y el control interno del proceso personal de aprendizaje" (1995, p.98), ¿qué será, entonces, la enseñanza? ¿Será independiente del aprendizaje? Si consideramos las diferentes formas en que una persona puede construir sus conocimientos, se podrían asumir tres tipos de 
aprendizaje: (a) por descubrimiento o aislado, que implica la interacción unísona del sujeto que aprende con la realidad o situación que le permite la creación de significados, principalmente producto de aciertos y desaciertos; (b) indirecto, en el que se pueden incluir el aprendizaje por imitación e, incluso, lo que algunos llaman procesos autodidácticos, donde la situación de aprendizaje se logra por observación de las manifestaciones subjetivas de otros o mediante la interacción con materiales de aprendizaje que otros hicieron para "enseñar", respectivamente; y (c) como proceso intersubjetivo, caracterizado por una compleja interacción dialógica entre dos o más sujetos, mediada o no por materiales, que les permite establecer juntos los significados correspondientes.

Aunque no pareciera, la enseñanza está implícita en cada una de estas definiciones. Pero, como proceso pedagógico de aula, que es el interés principal en este ensayo, la última planteada es la que más encaja. Es decir, la enseñanza, más que una representación clara de lo que se espera de los procesos expositivos o de instrucción, debe reconocerse conceptual y metodológicamente desde el significado del aprendizaje. No como un acto del sujeto que enseña, sino como un proceso dinámico que acompaña, provoca y realza inexorablemente el proceso de aprendizaje. Esto implica que el aprendizaje no es realmente una consecución de la enseñanza, sino que, ambos, más que procesos complementarios, son "remos de un mismo barco", sincrónicos, dirigidos a un mismo fin, el proceso formativo. Incluso, el docente yo no es el actor principal o ejecutor de la enseñanza, solo es un medio por el cual ésta puede manifestarse.

\section{3. ¿Predominio de la enseñanza tradicional o miedo al cambio? ¿Un nuevo rol para el docente?}

Una concepción diferente de lo que es la enseñanza y el aprendizaje, por supuesto, representa un choque a la cultura y habitualidad de un docente tradicional-expositivo. Especialmente, cuando existen elementos que se oponen a una visión diferente de ver y hacer la enseñanza. Muchos docentes pueden preguntarse, por ejemplo, ¿cómo crear espacios pedagógicos que permitan el aprendizaje de todos mis estudiantes "por igual" si debo abordar "todos los contenidos" que me impone el diseño curricular en el tiempo asignado? En esta pregunta resaltan tres aspectos básicos del currículo, o del sistema educativo, que muchas veces limitan o direccionan al docente en su forma de actuar en los procesos educativo-formativos, tales como: el tiempo, los contenidos, la modalidad de evaluación y las características propias de los estudiantes (como individuos y/o como grupo). 
Respecto a los contenidos, hay que tener claro que éstos por sí mismos no son el eje central de la enseñanza, pero sí juegan un papel importante. Pues, por un lado, están dotados de un significado sociocultural (Serrano y Pons, 2011), y representan el trabajo de un sin fin de personajes que se dedicaron a su construcción para la comprensión de nuestra realidad en momentos históricos y geográficos determinados, siendo, muchas veces, productos de controversias y consensos que se obvian en el proceso pedagógico. Por ello, no deben tratarse como verdades absolutas, y debe buscarse su contextualización con la propia realidad de los sujetos que aprenden.

Por otro lado, el juicio de la cantidad, complejidad y finalidad de estos contenidos, puede permitirle al docente decidir respecto a la profundidad, alcance y medios más apropiados para traerlos al escenario pedagógico y promover su aprendizaje en el tiempo de aula establecido. Ducret (2001), citando a Piaget, señalaba que lo más recomendable es "enseñar lo esencial y retrasar al máximo una especialización demasiado marcada" (p.159), favoreciendo más "el desarrollo de la inteligencia y de la cultura en general" (p.169). Esto podría entenderse como los procesos reflexivos que deben darse en los estudiantes sobre lo que ya saben, qué y cómo aprenden, así como los cambios que puedan estar ocurriendo en sus propias concepciones, de manera que reconozcan sus potencialidades y autonomía en y para el aprendizaje, es decir, se logre la metacognición.

¿Qué tan difícil es desligarse de las prácticas expositivas tradicionales para asumir otras que permitan dar un poco más de protagonismo al sujeto que aprende? ¿Por qué parece que el esquema tradicional tiene gran predominio en el actuar educativo, especialmente en el área de ciencias? Aunque muchas respuestas a esta pregunta podrían ser producto del prejuicio, sería interesante traer a colación una pequeña historia señalada por Benarroch (2010):

Un grupo de científicos encerró a cinco monos en una jaula, en cuyo centro colocaron una escalera y, sobre ella, un montón de plátanos. Cuando uno de los monos subía la escalera para agarrar los plátanos los científicos lanzaban un chorro de agua fría sobre los que se quedaban en el suelo. Pasado algún tiempo, los monos aprendieron la relación entre la escalera y el agua, de modo que cuando un mono iba a subir la escalera, los otros lo molían a palos. Después de haberse repetido varias veces la experiencia, ningún mono osaba subir la escalera, a pesar de la tentación de los plátanos. Entonces, los científicos sustituyeron a uno de los monos por otro nuevo. Lo primero que hizo el mono novato nada más ver los plátanos fue subir la escalera. Los 
otros, rápidamente, le bajaron y le pegaron antes de que saliera el agua fría sobre ellos. Después de algunas palizas, el nuevo integrante del grupo nunca más subió por la escalera. Un segundo mono fue sustituido, y ocurrió lo mismo con el que entró en su lugar. El primer sustituido participó con especial entusiasmo en la paliza al nuevo. Un tercero fue cambiado, y se repitió el suceso. El cuarto, y finalmente el quinto de los monos originales fueron sustituidos también por otros nuevos. Los científicos se quedaron con un grupo de cinco monos que, a pesar de no haber recibido nunca una ducha de agua fría, continuaban golpeando a aquél que intentaba llegar hasta los plátanos. Si fuera posible preguntar a alguno de ellos por qué pegaban con tanto ímpetu al que subía a por los plátanos, con certeza ésta sería la respuesta: «No lo sé. Aquí, las cosas siempre se han hecho así». (p. 11)

La analogía de esta historia con la educación, es más que obvia. Los docentes se han acostumbrado, y han acostumbrado a sus estudiantes, a una educación así, unidireccional. Incluso, aunque los docentes en su formación han tenido una aproximación a teorías y prácticas educativas más acordes, suelen verse influenciados indirectamente por los profesores que les "enseñaron" bajo esta postura (Furió y Gil, 1989). Por su parte, ese intento por homogeneizar el proceso de aprendizaje, desatendiendo a las individualidades (realidades particulares y tiempos personales de aprendizaje), da la ilusión de evitar o reducir las diatribas con el tiempo para abordar a cabalidad todos los contenidos del currículo. Y no se puede olvidar que esta postura también da la ilusión de "control" y/o "superioridad" que muchos docentes se rehúsan a perder.

Aun así, es notorio el esfuerzo de naciones, entes gubernamentales e institucionales que han insistido y trabajado en implementar cambios curriculares, los cuales no tienen valor alguno si no hay cambios en la praxis pedagógica del docente (Benarroch, 2010; Chamizo, 2001; Grundy, 1998). Es decir, los cambios solo serán posibles si los docentes son partícipes, reflexiva y activamente en ellos, revisando sus concepciones de lo que significa la enseñanza, el aprendizaje, el currículo e, incluso, la evaluación.

Por otro lado, tampoco hay que irnos al otro extremo, pues, ciertamente, son los estudiantes quienes reconstruyen el conocimiento (aprenden). Sin embargo, el docente también reconstruye, junto a ellos, ese saber. De ahí que no sea suficiente conocer sobre los contenidos del currículo y, de forma aislada, los aspectos pedagógicos. El docente debe valerse de sus capacidades para integrar estas cualidades, a la vez que requiere tener una 
idea de la realidad de los estudiantes (ideas previas), para así lograr, en la dinámica dialógica, darle significado a dichos contenidos (Driver, 1988; Lorenzo, 2012; Talanquer, 2004; von Glasersfeld, 2001; Weil-Baris, 2001).

Esto demanda que la dinámica de aula se desarrolle en un ambiente de confianza (no amenazante), donde el estudiante pueda expresarse libremente y que le permita a ellos mismos, desde la reflexión individual y conjunta (diálogo docente-estudiante, estudianteestudiante y estudiante consigo mismo), darse cuenta de sus equivocaciones $y$, particularmente, de cómo corregirse. Lograr que los estudiantes se reconozcan a sí mismos y a los demás como sujetos inacabados y en constante formación, es un punto clave para que realmente exterioricen sus subjetividades (ideas, experiencias, opiniones, visiones, sentimientos,...) en el proceso educativo.

El docente, en esta dinámica, ya no sería esa figura idealizada de conocimiento absoluto, pero sí un sujeto de mayor experiencia que puede orientar al estudiante a una cultura más acorde de interacción con el saber. Incluso, pueden y deben demostrar su inacabamiento, así como la importancia de "aprender" y formarse en día a día, con cada oportunidad, pero con responsabilidad y compromiso.

Los contenidos tampoco tendrían control absoluto sobre el actuar docente, ni sobre los estudiantes, ni siquiera sobre la dinámica de aula, pues ésta debe tener su propia autonomía y espontaneidad. El docente es iniciador de ésta, provocando el diálogo y la reflexión, a la vez que observa y reflexiona. Ciertamente esto no es una tarea fácil, pero es lo que debería suceder (Gavidia, 2005).

\section{Retos en la enseñanza de la química}

Considero difícil que un docente de química no inicie sus cursos manifestando que ésta es la ciencia que estudia la materia, y a riesgo de que esto suene presuntuoso, ¿acaso hay otra manera de decir qué es la química? Todo profesional que se ha formado en esta área del saber debe reconocer y hacer ver a sus estudiantes que esta ciencia tiene un campo de actuación amplio y fascinante. Una mejor comprensión de nuestra realidad, así como el desarrollo de nuevos materiales y tecnologías cada vez más poderosas y sorprendentes son solo una muestra del arduo trabajo de los químicos y del conocimiento construido por ellos a lo largo de la historia. Su interrelación con otras ciencias, como la física, la biología, la medicina, la ingeniería de materiales, entre otras, muestra porqué la química se ha posicionado como una ciencia central. Pero, ¿y su enseñanza? 
En el campo educativo la química parece no estar teniendo el éxito que se esperaría de una ciencia como ella, por el contrario, se habla de una crisis en su enseñanza (Benarroch, 2010; De Jong, 1996; Galagovsky, 2005; Garritz 2010; Izquierdo, 2004). ¿Cómo debemos asumir los docentes tal crisis?

Esta crisis en la enseñanza de la química obliga a prestar mayor atención a todos esos factores, manifiestos o no, que perturban el acto pedagógico. Esto incluye lo que ocurre en el aula (cómo son presentados los contenidos, los procesos cognitivos del estudiante, los procesos socio-dialógicos,...) y, muy especialmente, lo que pasa más allá de ésta (la cotidianidad del estudiante, la dinámica propia del conocimiento, la realidad en general,...). Por ello, a riesgo de dejar por fuera muchas realidades particulares e importantes, se hace énfasis en tres aspectos que, más que retos al enseñar química, son puntos clave que deben considerarse durante la praxis pedagógica en esta área del saber.

\subsection{Enseñar a comprender la química}

Entre los factores que afectan el proceso educativo en química, es evidente la dificultad que exteriorizan los estudiantes para comprender los conceptos y las resoluciones matemáticas dentro de la química, así como su articulación con las aproximaciones empíricas que se tienen del mundo real. Aunque no es un reto nuevo, se ha mantenido por mucho tiempo. De acuerdo con las ideas de Johnstone (1991, 2006), esta comprensión puede lograrse desde tres niveles de pensamiento distintos que se encuentran interrelacionados entre sí: un nivel macroscópico, al que se puede acceder a través de los sentidos y que nos permite un acercamiento a lo que conocemos como mundo real; uno submicroscópico, más imaginativo, al que se puede acceder solo mediante el pensamiento y que permite constituir las explicaciones teóricas de los fenómenos; y otro simbólico, que permite expresar y representar (principalmente, de forma escrita) los fenómenos a nivel macro y submicroscópico. Estos niveles o dominios han sido formulados bajo lo que se conoce como metáfora del triángulo equilátero (figura 1), en la que un químico o un docente de esta área podría ubicarse sin dificultad al estudiar un determinado fenómeno, incluso, al "explicarlo", bien en un vértice, una arista o algún punto dentro del triángulo. Pero, ¿y los estudiantes? 
Figura 1

Niveles de representación de la química

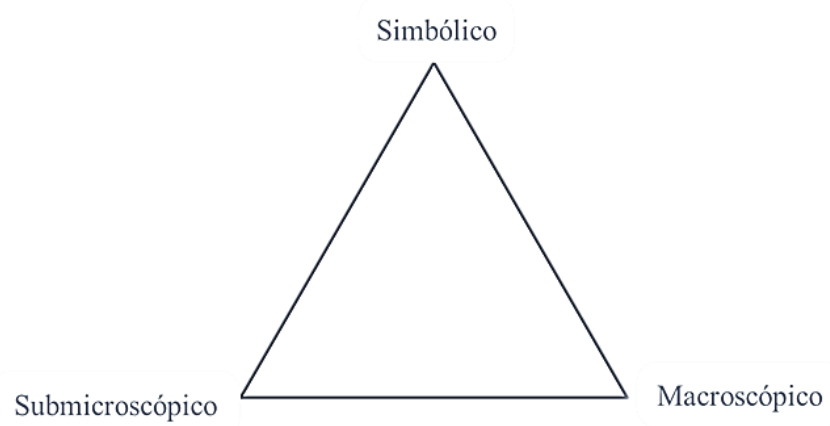

Fuente: Adaptación de Jhonstone $(1991,2006)$

Si adaptarse a estos tres niveles de pensamiento podría resultar complicado para el docente, más lo es para los estudiantes, principalmente cuando se enfrentan a nuevos contenidos. Incluso Jhonstone reconocía las limitaciones que presentan los estudiantes con el pensamiento multinivel, quien indicó que éstos, por lo general, tienden a estancarse en alguno de los vértices. De acuerdo con Benarroch (2010) el problema podría radicar en la poca distinción que se hace entre estos dominios descriptivos durante el proceso de enseñanza, el cual, por lo general, inicia desde el nivel simbólico (y en muchos casos se mantiene en éste). Pero, ¿cómo hacer tal distinción al enseñar química?

Aunque la actividad comunicativa-simbólica es una de las manifestaciones subjetivas del ser humano más desarrollada y útil, la actividad pedagógica no puede quedar estancada en el vértice simbólico, principalmente por parte del docente. Por ejemplo, una excelente oportunidad para abordar el dominio macroscópico de la química, ciertamente, son las actividades experimentales. Sin embargo, debemos evitar reducirlas a actividades de comprobación para refuerzo de las clases teóricas, pues podrían disminuir o perder su utilidad didáctica (Benarroch, 2010; Izquierdo, 2004; Nakamatsu, 2012). El acercamiento a la fase experimental de la química implica más que solo observar un fenómeno, ya que es una excelente oportunidad para que el estudiante conozca cómo los químicos trabajan y actúan ante esos fenómenos y, a la vez, cómo lo observable se explica y teoriza en conocimiento científico. Es decir, cómo se realiza la conexión o desplazamiento hacia los niveles submicroscópico y simbólico, ya que lo que se observa a nivel macroscópico es un reflejo de lo que ocurre a nivel submicroscópico, lo cual también debe representarse con herramientas lingüística (símbolos, ecuaciones químicas, cálculos matemáticos,...). 
Debido al carácter explicativo del nivel submicroscópico, se requiere mucha abstracción e imaginación. Quizá por ello, este nivel no es fácilmente comprendido por los estudiantes en muchos niveles educativos (Ordenes, Arellano, Jara y Merino, 2014). ¿No es acaso un reto pensar en algo ínfimamente pequeño que no se puede ver (como átomos, electrones, moléculas,...)? ¿Cómo imaginar, representar y manejar modelos explicativos y a la vez reconocer que realmente no es, no ha sido, y quizá nunca será así lo que plantean? Lograr reconocer la "ilusión" de los modelos, lo inacabado de las teorías y del conocimiento, es el reto. Pues, debemos recordar que la gran variedad de modelos que se plantean en la literatura no son más que la expresión simbólica de lo que pensaron algunos científicos (otras personas), por lo que se requiere de experiencia y reflexión para asimilar, no de modo estricto, tales representaciones. Eso es lo que los estudiantes deben aprender a reconocer, con la ayuda del docente.

Por ello, promover la comprensión de lo que tiene carácter explicativo y representacional, los modelos, implica una interconexión real con los otros dominios de pensamiento, tratando de evitar la generación de concepciones inadecuadas. Como señalaba Izquierdo (2004): "la docencia ha de facilitar que los estudiantes puedan establecer relaciones significativas entre las teorías químicas, las intervenciones experimentales que pueden llevar a cabo y los lenguajes con los que hablar de ellas" (p.124). Es decir, el docente en su praxis pedagógica debe ofrecer oportunidades a los estudiantes para que aprendan a reconocer cada uno de estos dominios (sin predominio de alguna) y justificar (o explicar) el porqué de ellos, al abordar los diferentes fenómenos químicos, tanto en el ámbito escolar como en su cotidianidad. Sin embargo, el señalamiento de crisis en la enseñanza de la química sugiere que aún hay deficiencias. ¿Será que muchos docentes de química aún no tienen esto claro?

Articulando un poco con lo discutido en la sección anterior, es seguro que una clase de química (o de cualquier otro curso) no se desarrolla exactamente igual a otra, especialmente si se trata de diferentes grupos de estudiantes, pues la naturaleza humana y socio-histórica hacen presencia inexorablemente dentro del acto educacional. Lo mismo ocurre con contenidos diferentes que se traen al escenario pedagógico con un mismo grupo. Por ello, no hay dinámicas universales o utópicas, lo que hace necesario una búsqueda responsable y constante para el actuar docente. La ventaja de los contenidos de química es que todo a nuestro alrededor puede verse desde esta ciencia. Así, el reto docente se centra en cómo los contenidos de química se convierten en posibilidades de aprendizaje para los estudiantes. 
Más aún, cuando lo que se percibe son estudiantes menos interesados en comprender los fenómenos que ocurren a su alrededor.

Lo ideal sería iniciar siempre una indagación de cuáles son esos fenómenos que pueden resultar de interés para los estudiantes. Pues, aquellos "fenómenos químicos que resultan familiares a los alumnos o semejantes a otros fenómenos familiares, [resultarían] fácilmente inteligibles y utilizables para el aprendizaje de la química" (De Manuel, 2004, p.30, cursiva original de la fuente). Además, como ha señalado Garritz (2011), la contribución que ha tenido la química en diferentes áreas tanto científicas como cotidianas (por ejemplo, alimentos, el origen de la vida, el genoma humano, medicamentos y salud, materiales, energía, el hoyo de ozono, sustentabilidad, la cocina y el deporte) puede y debe ser considerada en nuestras clases de química para una mejor comprensión de esta ciencia. De ahí que la formación continua y amplia del docente (científica, pedagógica, cultural, social,...) es clave en el acto educacional, haciendo más atractiva la actividad pedagógica desde una química más real e interesante.

Pero, ¿cómo se relaciona todo esto con la metáfora del triángulo? De acuerdo con Mahaffy (2004) la metáfora del triángulo debe adicionar otro vértice, el ser humano, para representar la interacción de la química con quienes la construyen (aprendiz, científico, sociedad en general) y con su entorno (ambiente, planeta, universo). Esto convierte el triángulo en un tetraedro (figura 2). Sin embargo, ¿no está ya el hombre implícito en cada uno de los vértices?, ¿quién aprecia la realidad?, ¿quién la imagina y modela a nivel atómico-molecular?, o ¿quién la describe e interpreta simbólicamente? Por ello, ese nuevo vértice podría interpretarse mejor como la significancia que ha tenido, tiene y tendrá la química para el hombre en el mundo.

Figura 2

Enseñanza de la química tetraédrica: un nuevo énfasis en el elemento humano
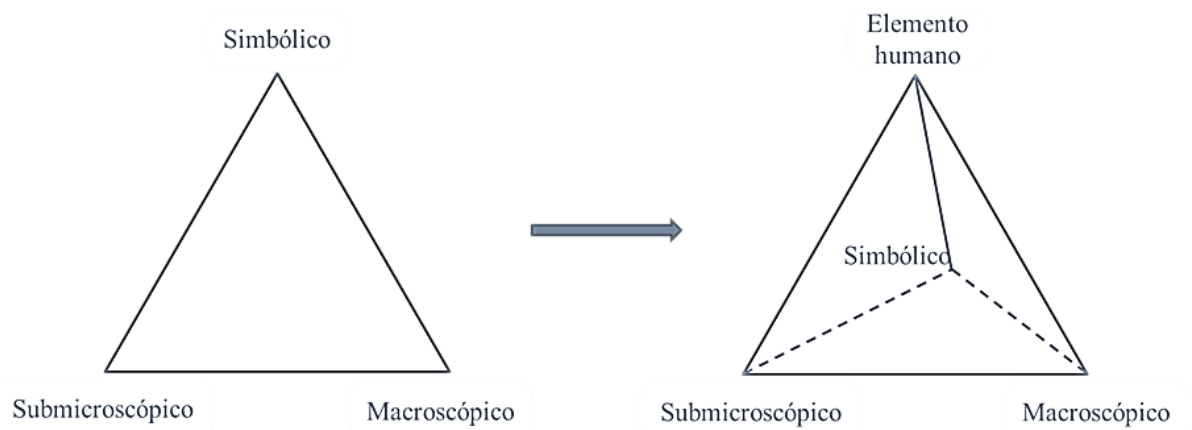

Fuente: Adaptado de Mahaffi (2004). 
Asumir explícitamente estos niveles representacionales en el proceso pedagógico de la química podría promover que un estudiante que inicie o continúe su experiencia en esta área de la ciencia no solo se haga consciente de estos niveles de pensamiento, sino que se sumerja con criticidad en la experiencia de ver, comprender, explicar, describir y reflexionar desde la química para sí mismo, su mundo y su yo con el mundo.

\subsection{Evitar una imagen inadecuada de la química}

Los estudiantes que van a iniciar un curso de química, por lo general, ya tienen una imagen preconcebida de esta ciencia como asignatura, que muchas veces, afecta sus posibilidades cognoscentes para ésta. Mora y Parga (2010), particularmente, describen tres imágenes de la química: (a) una académica, caracterizada por una visión estereotípica de la actividad científica (empirismo radical) y una sobrevaloración del conocimiento científico sobre otras formas de saber (racionalidad rigurosa); (b) una escolar, asociada a los currículos educativos, los textos escolares y las prácticas de enseñanza; y (c) una popular, generada por la interacción de la sociedad con los medios de comunicación, que promueven una reputación falsa de esta disciplina científica.

Todas ellas, sin embargo, parecen tener su epicentro en la poca experiencia de las personas con la química. Es decir, el conocimiento popular, de cierta manera, parece incluirse y complementar las otras dos. Incluso aquellos que han estudiado algo de química parecen mantener las ideas negativas que se tienen popularmente sobre esta ciencia. El colectivo o las instituciones no científicas, se han encargado de divulgar falacias que los químicos no se han enfocado fervientemente en desmentir (Edwards, Ceci y Ratcliffe, 2016; Mammino, 2001; Vivas-Reyes, 2009;), asociándose entonces la química como algo malo, no natural, que causa daños al organismo o problemas de contaminación. No con esto se niega que la química ha tenido su papel "oscuro" en contra del bienestar de la humanidad, pero, como han mencionado Vilches y Gil (2011, p. 4), "la lista de contribuciones [...] sería al menos igualmente larga que la de sus efectos negativos". ¿Por qué no aprovechar audazmente ambos aspectos en el proceso pedagógico?

Discutir por qué los químicos han llevado a cabo, o no, esto, o aquello, podría posibilitar la crítica, la reflexión e, incluso, la autorregulación en el acto educacional. Sería una iniciativa audaz para propiciar el diálogo, bajo el intercambio de ideas y posturas personales entre el docente y los estudiantes frente a esos problemas en el que la química ha tenido un papel causante o sanador, potenciándose el aspecto ético que se requiere en 
los nuevos tiempos (Matlin, Mehta, Hopf y Krief, 2016; Reibstein, 2017). Aprovechar acontecimientos reales o esas falacias que se difunden popularmente respecto a esta ciencia, implica traer al escenario educativo el contexto universal del conocimiento, desmontando la creencia de que éste solo está subordinado al ámbito académico o para "uso de los científicos", a la vez que posibilitaría ese aspecto importante de la enseñanza, casi perdido con el esquema transmisor-expositivo, la relación sujeto-conocimiento-mundo.

Casos como estos los encontramos en la propuesta de Torre-Fernández y GarcíaGranda (2012), quienes usaron el cine y la televisión como herramientas amenas y divertidas para generar discusiones sobre las "verdades científicas" que ahí se presentan; así como en la de Álvarez, López, Neira y Pazos, (2010), quienes propusieron como experiencia de laboratorio la recreación de un accidente marítimo que involucró la reacción entre el sodio y el agua.

La idea central de este tipo de propuestas es ofrecer oportunidades para favorecer el desarrollo del sentido crítico en el estudiante, a la vez que se plantea la búsqueda de esas bases científicas que le permitan confirmar eso que es noticia (lo que pasa en el mundo) o, en un sentido popperiano, falsear eso que resulta entretenimiento (lo que nos venden los medios). También podría ser necesario traer a colación, bien durante estas actividades dialógicas, o a través de trabajos prácticos, cómo los científicos trabajan y se enfrentan al conocimiento, derribando esa idea estereotípica del científico encerrado en su laboratorio haciendo experimentos secretos.

Hodson (1985) señalaba que esa visión distorsionada de la ciencia y del quehacer científico muchas veces se debe a la enseñanza expositiva, principalmente en el laboratorio, bajo una epistemología empirista-idealista, por lo que la praxis pedagógica debe reorientarse hacia enfoques epistemológicos más adecuados. Sin embargo, esta visión epistemológica sigue arraigada en muchos profesores de ciencia (Carrascosa, Gil, Vilches y Valdés, 2006). ¿Cómo cambiar estos enfoques epistemológicos? ¿Cuáles son los más adecuados?

Níaz (1994) ha planteado que una visión lakatosiana puede generar una visión más real de la naturaleza de la ciencia, donde el conocimiento no se construye de manera lineal y acumulativo, sino que se debe a la rivalidad y competencia de programas de investigación que permiten la transición epistémica de las teorías científicas hacia aquellas que tienen un mayor poder explicativo. Otra alternativa epistemológica es la que plantea la corriente constructivista, donde, 
...el conocimiento no es el resultado de una mera copia de la realidad prexistente [como sugieren las ideas positivistas], sino un proceso dinámico e interactivo a través del cual la información externa es interpretada y reinterpretada por la mente. En este proceso la mente va construyendo progresivamente modelos explicativos, cada vez más complejos y potentes, de manera que conocemos la realidad a través de modelos que construimos ad hoc para explicarla. (Serrano y Pons, 2011, p. 11)

Sin embargo, estos modelos, aunque muestran un mejor acercamiento a cómo se construye el conocimiento científico que una aproximación positivista, también parecen quedar encapsulados en el ámbito académico-escolar, protagonizado ahora no por los contenidos, sino en "el método" que le da origen. $\mathrm{Y}$, aunque permite resaltar el papel del científico como ser creador y transformador del conocimiento como individuo y como grupo de una comunidad epistémica, poco se enfatiza en la relación sujeto-conocimiento-mundo.

No se quiere decir con esto que estos planteamientos no han sido útiles o exitosos, por el contrario, la idea es superar las posibles deficiencias de estas propuestas con lo que ya se ha expuesto, articular más la química con la cotidianidad, con su papel en la sociedad, y la posibilidades epistemológicas y cognitivas que permiten comprenderla, construirla y aprovecharla.

\subsection{Superar los límites del currículo}

En los dos apartados anteriores se planteó la necesidad de ir más allá de los contenidos del currículo de química, en el sentido de contextualizarlos de acuerdo con las realidades a las que se exponen nuestros estudiantes día a día (la cotidianidad) y los acontecimientos de nuestro mundo, a modo de abordar cada uno de los niveles de pensamiento y mostrar una imagen más adecuada de esta ciencia. Como se ha mencionado, la química es una ciencia amplia cuyo campo de estudio es la materia y, de acuerdo con Talanquer (2009), tiende a dar respuesta a cuatro preguntas fundamentales (tabla 1). Este autor, plantea que estas preguntas son cruciales para comprender y prever el presente y futuro de la química, así como los de su enseñanza. 
Tabla 1

Aspectos principales que atiende la química

\begin{tabular}{|c|c|l|}
\hline Pregunta & Intensión & \multicolumn{1}{|c|}{ Características } \\
\hline ¿Qué es esto? & Análisis & $\begin{array}{l}\text { Se relaciona con el desarrollo de técnicas analíticas e } \\
\text { instrumentos para separar, detectar, identificar y cuantificar } \\
\text { sustancias presentes en un sistema, así como para } \\
\text { proporcionar claves sobre la composición atómica y geometría } \\
\text { molecular. }\end{array}$ \\
\hline ¿Cómo lo hago? & Síntesis & $\begin{array}{l}\text { Se refiere al interés por crear nuevas sustancias que } \\
\text { presenten propiedades definidas: fármacos, materiales } \\
\text { poliméricos y óptico-electrónicos, entre otros, los cuales han } \\
\text { tenido y tendrán un gran impacto en nuestras vidas. }\end{array}$ \\
\hline ¿Cómo lo cambio? & Transformación \\
\hline ¿Cómo lo explico? & $\begin{array}{l}\text { La química tiene como meta la inducción, control y } \\
\text { manipulación de los procesos físicos y químicos que implican } \\
\text { la transformación de la materia, que puede ocurrir en } \\
\text { diferentes escalas espaciales y temporales. }\end{array}$ \\
\hline Modelaje & $\begin{array}{l}\text { La generación de modelos submiscroscópicos sobre la } \\
\text { estructura de la materia y sus transformaciones ha sido crucial } \\
\text { en el desarrollo de la disciplina, en cuanto a la capacidad } \\
\text { predictiva y explicativa sobre las propiedades y procesos de } \\
\text { transformación de sustancias nuevas y conocidas. }\end{array}$ \\
\hline
\end{tabular}

Fuente: Adaptación propia de lo planteado por Talanquer (2009).

Si nos fijamos bien en estas cuatro preguntas, de ellas se derivan la mayoría de los contenidos curriculares de los diferentes cursos de química, en cualquiera de los niveles educativos. Sin embargo, dichos contenidos se encuentran encasillados en bloques independientes y consecutivos, o "peldaños" de conocimiento que deben ir superándose, con lo cual, podríamos estar desaprovechando oportunidades reales para lograr su comprensión.

Por ejemplo, muchos cursos básicos de química a nivel universitario inician por el nivel submicroscópico (¿cómo lo explico?), dando una aproximación sobre la estructura atómica, las propiedades periódicas y los enlaces químicos; seguidamente, se tratan los aspectos macroscópicos de la química (¿cómo lo cambio?), tales como la preparación de disoluciones, los aspectos estequiométricos de las reacciones químicas y la estructura de la materia (gases, sólidos y líquidos). Las preguntas “¿qué es esto?” y “¿cómo lo hago?” son tratados en cursos más especializados, o pueden incorporarse como contenidos suplementarios bajo la figura de "química de frontera". ¿Sería posible salirse de esta estructura y reordenarlos de manera más interconectada? ¿Hacer una química más integral? Quizá, eso podría evitar que los conocimientos pierdan significado, pues, como se indicó al inicio, los estudiantes parecen dejar en el pasado reciente esos "conocimientos" una vez que son usados en una evaluación, consecuencia del esquema tradicional.

Un ejemplo interesante en este punto es el libro Química, un proyecto de la American Chemical Society (ACS, 2005), donde, aunque hay separación de contenidos por capítulos 
como la mayoría de los libros de texto, éstos se tratan muy contextualizados e interrelacionados entre sí, abordándolos desde la cotidianidad y, principalmente, asociándolos con los procesos biológicos.

Desvanecer los límites internos del currículo podría, incluso, ser más difícil, porque los seres humanos concebimos todo (o la mayoría de las cosas) como procesos, etapas y/o evolución. Y los contenidos del currículo no escapan de esta linealidad, planteándose la superación o comprensión de un tema, para poder comprender el siguiente. Y no se trata de negar esta realidad, ni hacer mezclas abruptas y sin sentido, sino que esa separación no puede ser estricta, pudiendo incorporar elementos "avanzados" cuando se tratan los "básicos", o por el contrario abordar un tema específico con el cual se pueden explicar los aspectos "básicos" de la química.

\section{Consideraciones finales}

¿Son necesarios otros escenarios de enseñanza alejados de la verticalidad? Seguramente habrá docentes que consideren que no, porque todavía no han dado el primer y más grade paso, reconocer que el modelo tradicional de enseñanza ya no es suficiente. La ventaja (o comodidad) de la enseñanza tradicional es que el rol del docente y del estudiante ya están definidos. Por ello, salir de esa "zona de confort" a la que el sistema educativo tradicional nos ha acostumbrado e intentar nuevas y más enriquecedoras experiencias implicará nuevas dificultades. Pero, ¿cómo podrían plantearse estos nuevos escenarios? Esto dependerá de cada docente, pues no hay recetas únicas ni utópicas en la enseñanza. ¿Significará esto que debemos entrar en un constante juego de ensayo y error? Quizá sí.

Así como el conocimiento es inacabado y está en constante crecimiento, el docente de química debe estar en constante búsqueda, tanto para actualizarse en esta área, como de las herramientas pedagógicas que puede disponer para convertir su clase en un espacio más acorde para el aprendizaje y la formación. Es otras palabras: para lograr enseñar, hay que atreverse a aprender. ¿No pretenderá el docente seguir creyendo que ya aprendió todo lo que tenía que aprender para su praxis? Esto significa que el docente también debe reconocer sus necesidades formativas.

Renovarse y reinventarse, puede ser la nueva praxis, pues cada clase, cada grupo, cada nuevo día es una oportunidad de conocer, investigar y crecer junto a los estudiantes en la construcción del conocimiento, pues su formación y la de sus estudiantes van de la mano. La praxis pedagógica requerirá que el docente aproveche audazmente la realidad a nuestro 
alrededor, los recursos y avances tecnológicos disponibles, y que realmente dialogue y reflexione junto a los estudiantes la significancia de los contenidos y de su comprensión y construcción.

Además, las propias reflexiones que el propio docente haga de su praxis, pueden y deben ser comunicadas. Por ello, la invitación que se intenta plantear en este trabajo no es solo para reflexionar la práctica docente desde otras perspectivas y los problemas que hacen que la enseñanza de la química esté en crisis, sino que el docente también es investigador sobre su praxis, debe experimentar y reflexionar.. Así, podrán, luego, hacer su propia invitación.

\section{Referencias}

Álvarez Gallego, Alejandro. (2003). La pedagogía y las ciencias: historia de una relación. En Olga Lucía Zuluaga, Alberto Echeverri, Alberto Martínez, Humberto Quiceno, Javier Sáenz y Alejandro Álvarez, Pedagogía y epistemología (pp. 271-300). Bogotá: Magisterio.

Álvarez Muíña, Constantino, López Prada, María Elena, Neira González, Mercedes y Pazos Alfonso, Mariano. (2010). Química en contexto: un accidente químico y la reacción del sodio con el agua. Educació Química, 6(1), 37-42. doi: 10.2436/20.2003.02.46

Arias, Roberto y Peralta, Helga. (2011). La enseñanza, una puerta para la complejidad y la crítica. Estudios pedagógicos, 37(1), 293-302.

Benarroch Benarroch, Alicia. (2010). Aportes de la investigación en la enseñanzaaprendizaje de la química para afrontar los desafíos de la universidad del siglo XXI. Revista de Facultad de Bioquímica y Ciencias Biológicas, 14, 9-33. Recuperado de http://bibliotecavirtual.unl.edu.ar/publicaciones/index.php/FABICIB/article/view/867/127 $\underline{9}$

Carrascosa, Jaime, Gil Pérez, Daniel, Vilches, Amparo y Valdés, Pablo. (2006). Papel de la actividad experimental en la educación científica. Caderno Brasileiro de Ensino de Física, 23(2), 157-181.

Chamizo, José Antonio. (2001). El curriculum oculto en la enseñanza de la química. Educación química, 12(4), 194-198. Recuperado de http://www.joseantoniochamizo.com/pdf/educacion/articulos/010 Curriculo oculto ens enanza quimica.pdf

De Jong, Onno. (1996). La investigación activa como herramienta para mejorar la enseñanza de la química: nuevos enfoques. Enseñanza de las Ciencias, 14(3), 279-288. Recuperado de https://dialnet.unirioja.es/servlet/articulo?codigo=94858

De Manuel Torres, Esteban. (2004). Química cotidiana y currículo de química. Anales de Química, 100(1), 25-33. Recuperado de https://dialnet.unirioja.es/servlet/articulo?codigo=818836 
Driver, Rosalind. (1988). Un enfoque constructivista para el desarrollo del currículo en ciencias. Enseñanza de las ciencias, 6(2), 109-120.

Ducret, Jean-Jacques. (2001). Constructivismo: usos y perspectivas en la educación. Perspectivas: Revista trimestral de educación comparada, 31(2) ,157-169. Recuperado de: https://dialnet.unirioja.es/servlet/articulo?codigo=3071590

Edwards, Jon, Ceci, Chiara y Ratcliffe, Elisabeth. (2016). What the public really thinks about chemistry. Chemistry International, 38(3-4), 16-19. doi: https://doi.org/10.1515/ci-2016$\underline{3-406}$

Eschenhagen, María Luisa. (2007). Diversas consideraciones y aproximaciones a la noción de complejidad ambiental. Gestión y Ambiente, 10(1), 83-94. Recuperado de http://www.redalyc.org/service/redalyc/downloadPdf/1694/169419796006/1

Escribano González, Alicia. (1995). Aprendizaje cooperativo y autónomo en la enseñanza universitaria. Enseñanza \& Teaching, 13, 89-102. Recuperado de https://dialnet.unirioja.es/servlet/articulo?codigo=95708

Freire, Paulo. (2005). Pedagogía del oprimido. (2da edición). Buenos Aires: Siglo XXI.

Furió-Mas, Cales y Gil Pérez, Daniel. (1989). La didáctica de las ciencias en la formación inicial del profesorado: una orientación y un programa teóricamente fundamentados. Enseñanza de las ciencias, 7(3), 257-265.

Galagovsky, Lidia. (2005). La enseñanza de la química pre-universitaria: ¿Qué enseñar, cómo, cuánto, para quiénes? Química viva, 4(1), 8-22.

Garritz, Andoni. (2010). La enseñanza de la ciencia en una sociedad con incertidumbre y cambios acelerados. Enseñanza de las Ciencias, 28(3), 315-326. Recuperado de http://www.raco.cat/index.php/Ensenanza/article/viewFile/210803/353410

Garritz, Andoni. (2011). La celebración del Año Internacional de la Química: Las contribuciones de la química al bienestar de la humanidad. Educación química, 22(1), 2-7. Recuperado de http://www.scielo.org.mx/pdf/eq/v22n1/v22n1a1.pdf

Gavidia, Valentín. (2005). Los retos de la divulgación y enseñanza científica en el próximo futuro. Didáctica de las ciencias experimentales y sociales, 19(1), 91-102. Recuperado de http://redined.mecd.gob.es/xmlui/handle/11162/22428

Glasersfeld, Ernest von. (2001). El constructivismo radical y la enseñanza. Perspectivas: Revista trimestral de educación comparada, 31(2), 171-184. Recuperado de http://unesdoc.unesco.org/images/0012/001249/124945so.pdf

Grundy, Shirley. (1998). Producto o praxis del currículum. Madrid: Ediciones Morata.

Hodson, Derek. (1985). Philosophy of science, science and science education. Studies in Science Education, 12(1), 25-57. doi: https://doi.org/10.1080/03057268508559922 
Izquierdo Aymerich, Mercè. (2004). Un nuevo enfoque de la enseñanza de la química: contextualizar y modelizar. Anales de la Asociación Química Argentina, 92(4-6), 115136. Recuperado de http://www.scielo.org.ar/scielo.php?script=sci arttext\&pid=S036503752004000200013\&lng=es\&tlng=es

Johnstone, Alex. (1991). Why is science difficult to learn? Things are seldom what they seem. Journal of Computer Assisted Learning, 7(2), 75-83. doi: 10.1111/j.13652729.1991.tb00230.x

Johnstone, Alex. (2006). Chemical Education Research in Glasgow in Perspective. Chemistry Education Research and Practice, 7(2), 49-63. doi: 10.1039/B5RP90021B

López Calva, Martín. (2008). Formación humana y sociedad del conocimiento: de la ambigüedad a la complejidad. Revista Internacional de Ciencias Sociales y Humanidades SOCIOTAM, 18(1), 53-71. Recuperado de http://www.redalyc.org/pdf/654/65411190003.pdf

Lorenzo, María Gabriela. (2012). Los formadores de profesores: El desafío de enseñar enseñando. Profesorado: Revista de currículum y formación del profesorado, 16(2), 343-360. Recuperado de http://www.ugr.es/ recfpro/rev162COL3.pdf

Mahaffi, Peter. (2004). The future shape of chemistry education. Chemistry Education Research and Practice, 5(3), 229-245. doi: 10.1039/B4RP90026J

Mammino, Liliana. (2001). Algunas reflexiones sobre la imagen de la Química. Anales de Química, 97(2), 48-52.

Matlin, Stephen, Mehta, Goverdhan, Hopf, Henning y Krief, Alain. (2016). One-world chemistry and systems thinking. Nature Chemistry, 8(5), 393-398.

Molina, Víctor. (2006). Currículo, competencias y noción de enseñanza aprendizaje. Necesidad de una reformulación de nuestras concepciones sobre educación. Revista Proyecto Regional de Educación para América Latina y el Caribe, (3), 50-63.

Mora Penagos, William Manuel y Parga Lozano, Diana Lineth. (2010). La imagen pública de la química y su relación con la generación de actitudes hacia la química y su aprendizaje. Tecné, Episteme y Didaxis, 27(1), 67-93.

Nakamatsu, Javier. (2012). Reflexiones sobre la enseñanza de la química. Blanco \& Negro Revista sobre Docencia Universitaria, 3(2), 38-46.

Ordenes, Ruby, Arellano, Marcela, Jara, Roxana y Merino, Cristian. (2014). Representaciones macroscópicas, submicroscópicas y simbólicas sobre la materia. Educación química, 25(1), 46-55.

Pérez Gómez, Ángel. (2002). Un aprendizaje diverso y relevante. Cuadernos de pedagogía, 311, 66-70.

Reibstein, Rick. (2017). A more ethical chemistry. Current Opinion in Green and Sustainable Chemistry, 8(12), 36-44. 
Serrano, José Manuel y Pons Parra, Rosa María. (2011). El constructivismo hoy: enfoques constructivistas en educación. Revista electrónica de Investigación educativa, 13(1). Recuperado de http://redie.uabc.mx/vol13no1/contenido-serranopons.html.

Talanquer, Vicente. (2004). Formación docente: ¿Qué distingue a los buenos maestros de química? Educación química, 15(1), 52-58.

Talanquer, Vicente. (2009). Química: ¿Quién eres, a dónde vas y cómo te alcanzamos? Educación química, 20(extraord.), 220-226.

Torre-Fernández, Laura y García-Granda, Santiago. (2012). La química en el cine: ficción o realidad. Anales de Química, 108(1), 44-48.

Vilches, Amparo y Gil Pérez, Daniel. (2011). Papel de la química y su enseñanza en la construcción de un futuro sostenible. Educación química, 22(2), 103-116.

Vivas-Reyes, Ricardo. (2009). Filosofía de la química: un área ampliamente olvidada. Revista de la Academia Colombiana de ciencias exactas, físicas y naturales, 33(126), 125-128.

Weil-Barais, Annick. (2001). Los constructivismos y la didáctica de las ciencias. Perspectivas: Revista trimestral de educación comparada, 31(2), 197-208. 